ORIGINAL PAPER

\title{
Clinicopathologic analysis of isolated hematuria IN CHILD/ADOLESCENT AND ADULT PATIENTS
}

\author{
Guanglei $\mathrm{Chen}^{1}$, Jun Zhu ${ }^{2}$, MingHui Song ${ }^{1}$, Lu Ma ${ }^{1}$, Tao Pan ${ }^{1}$, Qi Yang ${ }^{1}$, \\ WENSHENG $Z_{\text {HANG }}{ }^{1}$
}

\begin{abstract}
${ }^{1}$ Department of Nephrology, Beidaihe Sanatorium of Beijing Military Area Command, Qinhuangdao, China ${ }^{2}$ Department of Medical Administration, Beidaihe Sanatorium of Beijing Military Area Command, Qinhuangdao, China
\end{abstract}

GuangLei Chen and Jun Zhu contributed equally to this work and should be considered co-first authors.

\begin{abstract}
To our knowledge, no in-depth clinicopathologic study of isolated hematuria (IH) is currently available. To address this gap, we analyzed the clinicopathologic features of $\mathrm{IH}$ as it manifests in child/adolescent and adult patients. The clinical data and pathological types of $543 \mathrm{IH}$ patients who underwent renal pathological examinations from January 2005 to June 2010 were retrospectively analyzed. Clinical manifestations differed among the age groups: children/adolescents exhibited the highest percentage of mesangial proliferative glomerulonephritis $(41.78 \%)$, whereas adults showed the highest percentage of immunoglobulin A nephropathy (IgAN) (52.39\%). In addition, the percentage of IH patients who were classified according to clinical pathology differed from that of patients who were classified according to renal pathological type. Patients with IgAN who were found to have minimal proteinuria had more severe IH. For IH patients, especially those with a small amount of proteinuria, renal biopsy should be performed as early as possible in order to develop a long-term treatment plan and prognosis evaluation.
\end{abstract}

Key words: isolated hematuria, clinical, pathology, child, adolescent, adult.

\section{Introduction}

Isolated hematuria (IH) refers to renal disease that is clearly diagnosed as glomerular hematuria. IH patients exhibit elevated red blood cell (RBC) counts and no other clinical symptoms or changes during renal function tests $[1,2,3]$. IH can manifest as paroxysmal gross hematuria or persistent microscopic hematuria, which is not accompanied by edema, hypertension, or renal insufficiency $[4,5]$. The etiology and pathogenesis of these two forms of $\mathrm{IH}$, as well as their treatment and prognosis, are very different [4, 5].

With the improvement in general wellness and the popularity of urine tests, the number of $\mathrm{IH}$ pa- tients has also increased. An epidemiological survey of 6,311 urban cases (patients $>20$ years old) in Guangzhou, China, showed that hematuria has an incidence rate of $3.8 \%$ (95\% confidence interval [CI]: 3.4\%, 4.3\%) [6]. Vivante et al. [7] performed a 22-year follow-up assessment of cases of isolated microscopic hematuria among 3,690 Israeli adolescents and discovered that $0.7 \%$ of patients progressed to end stage renal disease (ESRD). Only $0.045 \%$ of non-microscopic hematuria patients also progressed to ESRD, so persistent asymptomatic microscopic hematuria in adolescent populations was considered an independent risk factor for ESRD. Thus, prompt treatment should be a cornerstone of the treatment approach in the wake of an $\mathrm{IH}$ diagnosis. However, 
very few studies have focused on IH. In fact, no indepth study of IH clinicopathologic features has been conducted to date.

In this article, clinically diagnosed $\mathrm{IH}$ patients underwent renal biopsy examination to further reveal the characteristics and course of IH. The clinicopathologic features in children and adult patients were then compared and analyzed.

\section{Material and methods}

A total of 543 patients were diagnosed with IH and underwent renal pathological examinations from January 2005 to June 2010 in our hospital. The patients were aged between 1.1 years old and 77 years old (mean age $29.72 \pm 15.91$ years old). The patients included 277 males and 266 females, with a maleto-female ratio of $1.04: 1$. This study was conducted in accordance with the Declaration of Helsinki. This study was conducted with approval from the Ethics Committee of Beidaihe Sanatorium of Beijing Military Area Command. Written informed consent was obtained from all participants.

\section{Inclusion and exclusion criteria}

The counting of centrifuged urinary RBCs $>3 / \mathrm{HP}$ and/or urinary sediment $\mathrm{RBC}>8 \times 10^{3} / \mathrm{ml}$ was set as the diagnostic criteria for hematuria. The inclusion criteria were as follows: 1) urinary test results had to meet the above diagnostic criteria two or more times, and 2) urinary samples had to exhibit severely deformed RBC (e.g., ring-like appearance, presence of spores or perforation) $\geq 30 \%$ or multiple malformed RBC $\geq 75 \%$. Exclusion criteria were as follows: 1) 24-hour urinary protein $\geq 0.3 \mathrm{~g}$;2) serum creatinine $\geq 132.6 \mu \mathrm{mol} / \mathrm{l}$; 3) edema; 4) abnormal blood pressure; 5) immunological abnormalities, including blood anti-O chain, complementary $\mathrm{C} 3$, HBsAg, HCVAb, or autologous antibodies; 6) secondary renal diseases, including diabetic nephropathy, lupus nephritis, or purpura nephritis; 7) diseases exhibited in the family history such as Alport syndrome; or 8 ) urinary calculus, urinary tuberculosis, hypercalciuria, urinary tract malformation, urinary tract cancer, left renal vein entrapment syndrome-induced hematuria, or other physiological urinary abnormalities. Indications of renal biopsy were as follows: 1) history of microscopic hematuria $\geq 6$ months and gross hematuria sustained more than half a month or reoccurring twice, and 2) the cause could not be confirmed by a conservative screening method.

\section{Clinical test methods}

Blood pressure measurements and physical examinations were performed routinely. Laboratory tests included a routine urine examination, urinary sedi- ment analysis, quantitation of 24-h urinary protein, urine three-cup test with urinary RBC morphology, serum $\mathrm{C} 3, \mathrm{C} 4, \mathrm{CH} 50$, anti-O chain evaluation, serum protein electrophoresis, blood urea nitrogen (BUN), urinary tract ultrasound, and analysis of serum creatinine, serum electrolytes, autologous antibodies, and serum markers of hepatitis B and C. The microscopic examination of urinary RBCs was performed as follows: the middle segment of fresh morning urine was obtained and then mixed and placed in a $10-\mathrm{ml}$ centrifuge tube for centrifugation at $1,500 \mathrm{rev} / \mathrm{min}$ for $5 \mathrm{~min}$. The sediment was approximately $0.5 \mathrm{ml}$. The amounts of urinary RBCs and abnormal RBCs in $1 \mathrm{ml}$ of urine were determined to calculate the percentage of abnormal RBCs. Each patient was made to undergo urinary RBC examination at least twice, and two examiners evaluated each specimen at least twice. A urinary sediment RBC count was performed toward the middle segment of the fresh morning urine with a UF-100i Automated Urine Particle Analyzer (Sysmex Corporation, Kobe, Japan).

\section{Pathological examination}

Methods included light microscopy and immunofluorescence, and certain individual cases underwent electron microscopic examination. The specimens for light microscopy included 10 or more renal glomeruli that underwent paraffin embedding. The slice thickness was $\leq 3 \mu \mathrm{m}$, and hematoxylin and eosin (HE), periodic acid-Schif (PAS), periodic Schiff-methenamine (PASM) and Masson's staining were performed. Patients with kidney damage caused by suspected ingredients underwent special staining (i.e., Congo red staining); the frozen sections were used for immunofluorescence. Fluorescein isothiocyanate-labeled, goat anti-human IgG, IgA, IgM, C3, C4, C1q, and Fib were used for direct immunofluorescence. Finally, the biopsy specimens of individual cases based on the requirements of the clinical diagnosis were sent for transmission electron microscopic examination at the Center of Electron Microscopy at the Third Hospital of Peking University. Observations included the thickness and structure of glomerular basement membrane, electron-dense materials and their deposition sites, morphology and cell inclusion of foot processes of glomerular epithelial cells, and anomalous fiber-like substances and virus-like particles.

\section{Pathological diagnosis}

According to the World Health Organization's classification of glomerular disease (1995) [8], pathological diagnosis should be divided into glomerular mild lesions (GML), mesangial proliferative glomerulonephritis (MsPGN), and endocapillary proliferative glomerulonephritis (EnPGN). In our study, GML ex- 
hibited no obvious lesions under a light microscope, or only mild mesangial hypercellularity, and negative or weakly positive immunofluorescence; electron microscopy showed no characteristic lesions. MsPGN light microscopy revealed that the mesangial cells and matrix exhibited mild, moderate, or severe diffuse hyperplasia, while immunofluorescence revealed IgG and complement C3 depositions in different mesangial areas with different intensities. These depositions were sometimes associated with the inner portion of the basal membrane; electron microscopy revealed mesangial proliferation associated with the deposition of low-density electron-dense materials. EnPGN light microscopy revealed diffuse proliferation of endothelial cells and mesangial cells, and immunofluorescence revealed IgG deposition and complementary $\mathrm{C} 3$, as coarse granules, along the outer side of the basal membrane; these were sometimes associated with depositions in the mesangial area. Electron microscopy revealed the proliferation of endothelial cells and mesangial cells, as well as camel hump-like, electron-dense deposits outside of the basal membrane; electron-dense material deposits could be seen in the mesangial areas (Fig. 1).

Among the three types of glomerular disease, the pathological changes of IgAN were analyzed and divided into classes I to $\mathrm{V}$ based on Lee's grading standards (Table I) [9]. The diagnostic criteria for MsPGN referred to in the literature were divided into mild (i.e., the proliferating mesangial cells and/or mesangial matrix did not exceed the capillary diameter), moderate (i.e., the proliferating mesangial cells and/ or mesangial matrix exceeded the capillary diameter, and the capillary lumen was squeezed) or severe (i.e., the proliferating mesangial cells and matrix had destroyed the capillary loops, initiating a segmental sclerosis state). In addition, several cases of thin basal membrane nephropathy and membranoproliferative nephritis were noted; these cases were rare, and thus were included with other classifications.

\section{Grouping}

Age groupings included: 1) child/adolescent ( $<18$ years old when treated): 146 cases, or 2 ) adult (aged $\geq 18$ years old when treated): 397 cases. Clin-
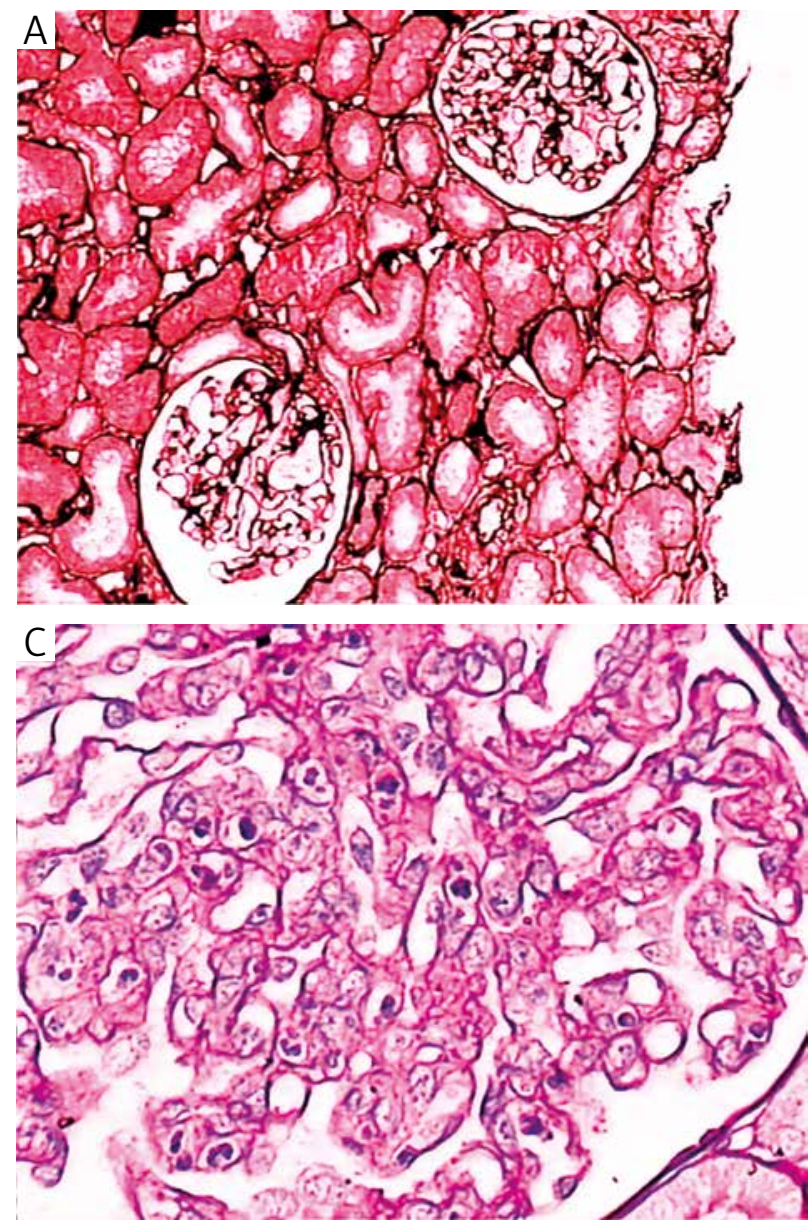
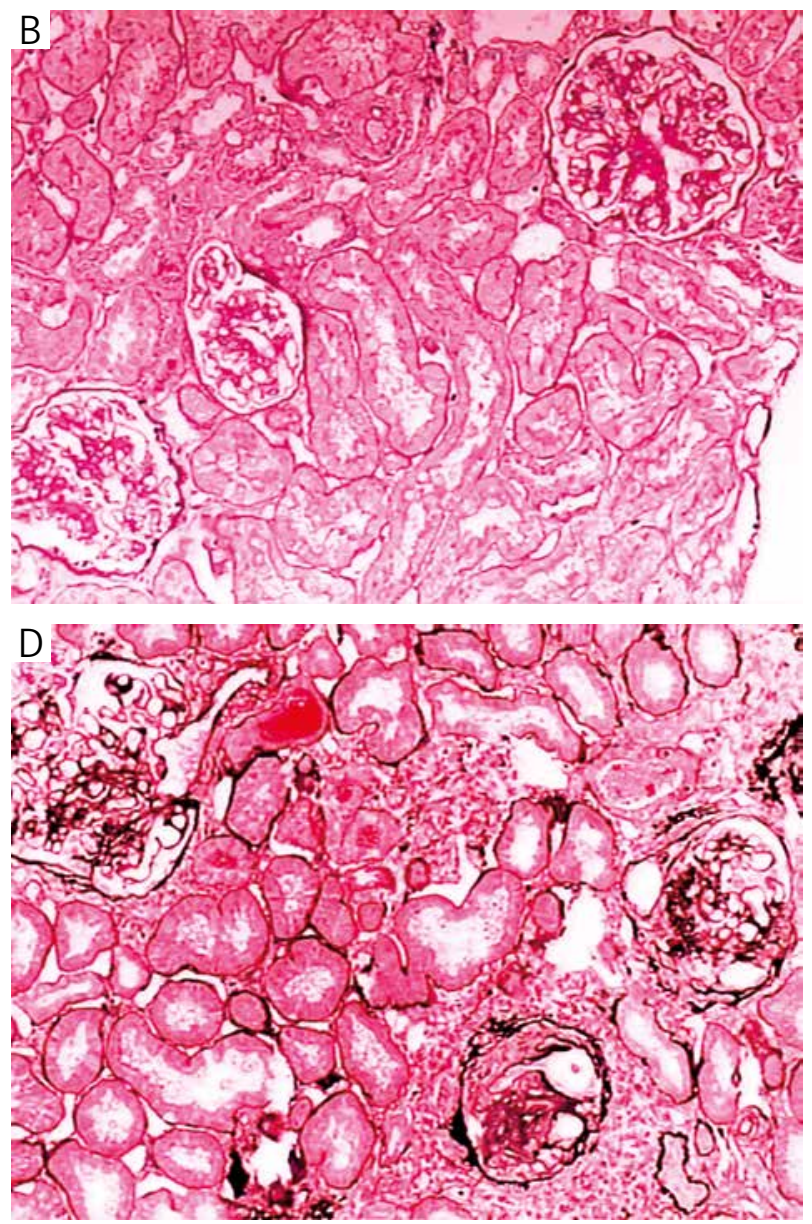

Fig. 1. Pathological figures and explanation of IgAN. A) mild lesions in renal glomerulus (PASM, 100×); B) mesangial proliferative glomerulonephritis (PAS, $100 \times$ ); C) endocapillary proliferative glomerulonephritis (PAS, $400 \times$ ); D) local proliferative sclerotic IgA nephrosis (level Lee IV) (PASM, 100×) 
Table I. Lee 's classification of IgAN

\begin{tabular}{clll}
\hline $\begin{array}{c}\text { Classifi- } \\
\text { CATiOn }\end{array}$ & Renal glomerulus & Renal tubule & Renal INTERSTItium \\
\hline I & Normal & No lesion & No lesion \\
\hline II & $\begin{array}{l}\text { Less than half of glomerular mesangial cells and matrix } \\
\text { exhibited mild hyperplasia or focal segmental sclerosis }\end{array}$ & No lesion & No lesion \\
\hline III & $\begin{array}{l}\text { Mesangial cells and matrix exhibited diffuse mild to moder- } \\
\text { ate hyperplasia, the focal segmental sclerosis increased, and } \\
\text { balloon adhesions and small crescents could be occasionally } \\
\text { seen }\end{array}$ & $\begin{array}{l}\text { Focal renal tubular } \\
\text { atrophy }\end{array}$ & $\begin{array}{l}\text { Edema in focal renal } \\
\text { interstitium and } \\
\text { inflammatory cell infil- } \\
\text { tration could be seen }\end{array}$ \\
\hline IV & $\begin{array}{l}\text { Mesangial cells and matrix exhibited diffuse moderate and } \\
\text { severe hyperplasia, 45\% or less of glomerular cells exhibited } \\
\text { crescents and hardening }\end{array}$ & $\begin{array}{l}\text { Multifocal tubular } \\
\text { atrophy }\end{array}$ & $\begin{array}{l}\text { Multifocal inflammato- } \\
\text { ry cell infiltration } \\
\text { in renal interstitium }\end{array}$ \\
\hline V & $\begin{array}{l}\text { Similar to class IV, while much more severe, or more than } \\
\text { 45\% of glomerular cells exhibited crescents }\end{array}$ & $\begin{array}{l}\text { Similar to class IV, } \\
\text { but much more } \\
\text { severe }\end{array}$ & $\begin{array}{l}\text { Similar to class IV, but } \\
\text { much more severe }\end{array}$ \\
\hline
\end{tabular}

ical groupings included: 1) microscopic and gross hematuria (MGH): 171 cases exhibiting microscopic hematuria and/or gross hematuria (quantitation of 24-h urinary protein was $<0.15 \mathrm{~g}$ ), or 2) small amount of proteinuria (SAP): 372 cases (quantitation of 24-h urinary protein, 0.15-0.3 g) with accompanying microscopic hematuria and/or gross hematuria. Pathological grouping was based on the type of pathological diagnosis and Lee's IgAN classification.

\section{Statistical analysis}

All statistical processes were performed with SPSS version 19.0 (IBM Corp., Armonk, NY, USA). Measurement data are represented as $\bar{x} \pm s$, with the non-parametric data represented by the median and the counting data expressed as the percentage. The intergroup comparisons of the measurement data were determined with $\mathrm{t}$ or rank sum tests, whereas the intergroup comparison of the counting data was determined by the $\chi^{2}$ test. Pearson test, the Yates correction method, or Fisher's exact test was selected according to the specific data, with $P<0.05$ considered as statistically significant.

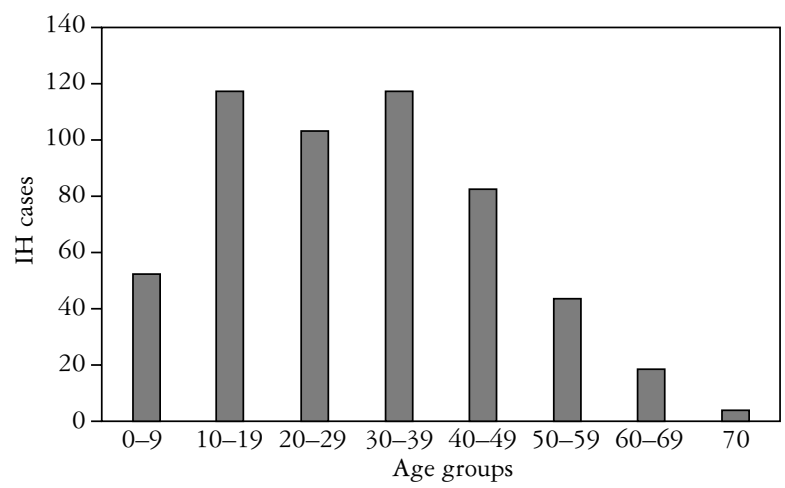

Fig. 2. IH cases of different age groups

\section{Results}

\section{Clinical manifestations}

The IH percentage was calculated according to the age groupings. The percentage of 10- to 39-yearold patients was higher, accounting for $62.61 \%$ of the sample size (Fig. 2), and there was a higher percentage of male patients when the child/adolescent and adult groups were compared. The medians of disease duration for the two groups (i.e., from onset to renal biopsy) were clearly different at 2.5 months (0.4 months to 79.5 months) and 7.1 months (0.5 months to 251.4 months), respectively. The hematuria percentage among children/adolescents being treated for respiratory tract infections was higher than that of the adult group, whereas more adults were diagnosed with $\mathrm{IH}$ when they had lumbar symptoms and during the course of their normal physical exanimations. No difference in the incidence rate of gross hematuria was found between the age groups, but the adult group exhibited a higher proportion of SAP than the children/adolescents (Table II).

\section{Pathological characteristics}

The renal pathological type of the child/adolescent group differed from that of the adult group, with the children/adolescents having the highest percentage of MsPGN. The pathological types of GML, MsPGN, and EnPGN were much more common in the child/ adolescent group, whereas the IgAN percentage was the highest and more common in the adult group (Table III).

\section{Clinical aspects and pathology}

Differences were observed in the renal pathological constituent percentage among the different clinical groupings of $\mathrm{IH}$ patients. For example, the MsPGN 
Table II. Clinical manifestations of IH in different age groups

\begin{tabular}{lcccc}
\hline Clinical manifestations & Child/adolescent $(\%)$ & Adult $(\%)$ & $\chi^{2}$ & P \\
\hline Cases & 146 & 397 & - & - \\
\hline Age at diagnosis (years) & $10.78 \pm 3.74$ & $36.69 \pm 12.66$ & - & 0.009 \\
\hline Gender ratio (M : F) & $88: 58=1.52: 1$ & $189: 208=0.91: 1$ & 6.854 & 0.000 \\
\hline Duration (months) & $2.5(0.4 \sim 79.5)$ & $7.1(0.5 \sim 251.4)$ & - & 0.004 \\
\hline Causes of discovery & & & & 0.529 \\
\hline Respiratory infection & $23(15.75 \%)$ & $30(7.56 \%)$ & 8.142 & 0.001 \\
\hline Urinary tract infection & $2(1.37 \%)$ & $11(2.77 \%)$ & 0.397 & 0.001 \\
\hline Lower back discomfort & $1(0.68 \%)$ & $36(9.07 \%)$ & 11.814 & 11.164 \\
\hline Normal physical examination & $12(8.22 \%)$ & $81(20.40 \%)$ & 0.382 & 0.536 \\
\hline Gross hematuria & $17(11.64 \%)$ & $39(9.82 \%)$ & 5.275 & 0.022 \\
\hline Accompanied by SAP & $89(60.96 \%)$ & $283(71.28 \%)$ & & \\
\hline
\end{tabular}

Table III. Pathological features of different age groups of IH patients

\begin{tabular}{|c|c|c|c|c|c|c|c|c|}
\hline GROUPING & $\mathbf{N}$ & GML & MsPGN & IGAN & ENPGN & OTHERS & $\chi^{2}$ & $\mathbf{P}$ \\
\hline Child/adolescent (\%) & 146 & $15(10.27)$ & $61(41.78)$ & $48(32.88)$ & $16(10.96)$ & $6(4.11)$ & 35.665 & 0.000 \\
\hline Adult $(\%)$ & 397 & $19(4.79)$ & $149(37.53)$ & $208(52.39)$ & $7(1.76)$ & $14(3.53)$ & & \\
\hline
\end{tabular}

Table IV. Clinicopathologic features of IH patients

\begin{tabular}{|c|c|c|c|c|c|c|c|c|}
\hline GROUPING & $\mathbf{N}$ & GML & MsPGN & IGAN & ENPGN & OTHERS & $\chi^{2}$ & $\mathbf{P}$ \\
\hline MGH group (\%) & 171 & $12(7.02)$ & $84(49.12)$ & $64(37.43)$ & $7(4.09)$ & $4(2.34)$ & 13.511 & 0.009 \\
\hline SAP group (\%) & 372 & $22(5.91)$ & $126(33.88)$ & $192(51.61)$ & $16(4.30)$ & $16(4.30)$ & & \\
\hline
\end{tabular}

MGH - microscopic and gross hematuria; SAP - a small amount of proteinuria; GML - glomerular mild lesions; MsPGN - mesangial proliferative glomerulonephritis; IgAN - IgA nephropathy; EnPGN - endocapillary proliferative glomerulonephritis nephritis

Table V. Clinicopathologic characteristics of different age groups of IH patients

\begin{tabular}{|c|c|c|c|c|c|c|c|c|c|}
\hline AGE GROUP & GROUPING & $\mathrm{N}$ & GML & MsPGN & IGAN & ENPGN & OTHERS & $\chi^{2}$ & $\mathbf{P}$ \\
\hline \multirow[t]{2}{*}{ Child/adolescent } & $\mathrm{MGH}(\%)$ & 57 & $4(7.02)$ & $32(56.14)$ & $15(26.32)$ & $5(8.77)$ & $1(1.75)$ & 8.474 & 0.076 \\
\hline & $\operatorname{SAP}(\%)$ & 89 & $11(12.36)$ & $29(32.58)$ & $33(37.08)$ & $11(12.36)$ & $5(5.62)$ & & \\
\hline \multirow[t]{2}{*}{ Adult } & MGH (\%) & 114 & $8(7.02)$ & $52(45.61)$ & $49(42.98)$ & $2(1.76)$ & $3(2.63)$ & 7.514 & 0.111 \\
\hline & SAP $(\%)$ & 283 & $11(3.89)$ & $97(34.28)$ & $159(56.17)$ & $5(1.77)$ & $11(3.89)$ & & \\
\hline
\end{tabular}

MGH - microscopic and gross hematuria; $S A P$ - a small amount of proteinuria; GML - glomerular mild lesions; MsPGN - mesangial proliferative glomerulonephritis; IgAN - IgA nephropathy; EnPGN - endocapillary proliferative glomerulonephritis nephritis

Table VI. Clinicopathologic features of MsPGN in children/adolescents and adults

\begin{tabular}{lccccccc}
\hline \multicolumn{1}{c}{ Age group } & $\begin{array}{c}\text { Clinical } \\
\text { Grouping }\end{array}$ & N & Mild MsPGN & $\begin{array}{c}\text { Moderate } \\
\text { MsPGN }\end{array}$ & $\begin{array}{c}\text { Severe } \\
\text { MsPGN }\end{array}$ & $\chi^{2}$ & P \\
\hline Child/adolescent & MGH $(\%)$ & 32 & $32(100.00)$ & $0(0.00)$ & $0(0.00)$ & 4.724 & 0.094 \\
\cline { 2 - 6 } & SAP $(\%)$ & 29 & $25(86.21)$ & $3(10.34)$ & $1(3.45)$ & & \\
\hline \multirow{2}{*}{ Adult } & MGH $(\%)$ & 52 & $45(86.54)$ & $6(11.54)$ & $1(1.92)$ & 4.289 & 0.117 \\
\cline { 2 - 6 } & SAP $(\%)$ & 97 & $71(73.20)$ & $17(17.53)$ & $9(9.27)$ & & \\
\hline
\end{tabular}

\footnotetext{
$M G H$ - microscopic and gross hematuria; $S A P$ - a small amount of proteinuria; $M_{s} P G N$ - mesangial proliferative glomerulonephritis
} 
Table VII. Clinicopathologic features of $\operatorname{IgAN}$ in children/adolescents and adults

\begin{tabular}{|c|c|c|c|c|c|c|c|c|c|}
\hline AgE GROUP & $\begin{array}{l}\text { CLINICAL } \\
\text { GROUPING }\end{array}$ & $\mathbf{N}$ & $\begin{array}{c}\text { GRADE I } \\
\text { IGAN }\end{array}$ & $\begin{array}{c}\text { GRADE II } \\
\text { IGAN }\end{array}$ & $\begin{array}{c}\text { GRADE III } \\
\text { IGAN }\end{array}$ & $\begin{array}{c}\text { GRADE IV } \\
\text { IGAN }\end{array}$ & $\begin{array}{c}\text { GRADE V } \\
\text { IGAN }\end{array}$ & $\chi^{2}$ & $\mathbf{P}$ \\
\hline \multirow[t]{2}{*}{ Child/adolescent } & MGH (\%) & 15 & $0(0.00)$ & $14(93.33)$ & $1(6.67)$ & $0(0.00)$ & $0(0.00)$ & \multirow[t]{2}{*}{2.771} & \multirow[t]{2}{*}{0.428} \\
\hline & $\operatorname{SAP}(\%)$ & 33 & $1(3.03)$ & $24(72.73)$ & $7(21.21)$ & $1(3.03)$ & $0(0.00)$ & & \\
\hline \multirow[t]{2}{*}{ Adult } & MGH (\%) & 49 & $2(4.08)$ & $30(61.22)$ & $12(24.49)$ & $5(10.21)$ & $0(0.00)$ & \multirow[t]{2}{*}{17.650} & \multirow[t]{2}{*}{0.001} \\
\hline & $\operatorname{SAP}(\%)$ & 159 & $1(0.63)$ & $52(32.70)$ & $75(47.17)$ & $28(17.61)$ & $3(1.89)$ & & \\
\hline
\end{tabular}

Note: MGH: microscopic and gross hematuria, SAP: a small amount of proteinuria, $\operatorname{Ig} A N$ : $\operatorname{Ig} A$ nephropathy

percentage in the MGH group was higher than that of the SAP group, whereas the IgAN percentage in the former was lower than that in the latter (Table IV). Regardless of age of the IH patients, no difference was observed in the renal pathological constituent percentage among the MGH groups when the patients were grouped according to different clinical manifestations (Table V).

In terms of the pathological changes in MsPGN and IgAN, cases of mild MsPGN exhibited the highest percentage of changes. No differences were generally found in the extent of MsPGN lesions among the different clinical groups (Table VI). Lee grade II had the highest percentage among the different clinical groups of IgAN in children/adolescents, whereas no difference was generally observed in Lee's classification among the different clinical groups. For the cases of adult IgAN, the highest Lee grade II was found in the MGH group and the highest Lee grade III was found in the SAP group. A comparison between the two groups showed that Lee grades I and II in the MGH group were higher than those in the SAP group, whereas Lee grades III and IV in the MGH group were lower than those in the SAP group (Table VII).

\section{Discussion}

Our study showed that 10- to 39-year-old patients accounted for $62.61 \%$ of the total cases of $\mathrm{IH}$. The percentage of patients older than 40 years old was remarkably decreased, which might be related to age-related changes in the renal-specific disease spectrum and other factors. However, the lack of epidemiological investigation results did not directly reflect the incidence within this age range. When grouped by age, the child/adolescent group had a higher percentage of males than the adult group, probably because the IgAN percentage in male IH children was higher than in females [10]. The duration medians of the two groups (i.e., from onset to renal biopsy) were clearly different at 2.5 months $(0.4$ months to 79.5 months) and 7.1 months (0.5 months to 251.4 months), respectively, because the children/adolescents tended to visit the hospital earlier and a higher degree of attention was paid to their symptoms. The hematuria percentage among children/adolescents hospitalized for respiratory infection was higher than that of adults, whereas more adults were diagnosed when they went to the hospital for lumbar symptoms and normal physical examinations. These results indicate the need for vigilance regarding clinical manifestations of IH and prodromal symptoms in different age groups. Medical examinations are especially important for adults who receive an early IH diagnosis. No difference in the incidence of gross hematuria was found between the different age groups, whereas the SAO percentage in the adult group was higher than that in the child/adolescent group. Through further clinical and pathological analyses, we revealed the pathological basis of this clinical feature.

The pathological appearances of $\mathrm{IH}$ are diverse. Zhang and Shen [11] reported that thin basement membrane nephropathy (TBMN), IgAN, MsPGN are the three most common renal pathologies in children diagnosed with IH. Elsewhere, Mo and Chen [12] reported that the renal pathological result order was most commonly MsPGN, ML, and IgAN. A recent study [5] showed that GML accounts for most pathological types of $\mathrm{IH}$ in children, followed by IgAN, TBMN, and MsPGN. Hall et al. [13] reported the biopsy results of 89 adult cases of asymptomatic microscopic hematuria; TBMN, IgAN, GML, and normal kidney tissue accounted for $43 \%, 20 \%, 19 \%$, and $18 \%$ of cases, respectively, which is similar to the percentages seen for the pathological constituents of children's IH.

Our study shows that pathological types differ among age groups. MsPGN was the most common in the child/adolescent group, followed by IgAN, EnPGN, and GML. By contrast, IgAN accounted for more than $50 \%$ of $\mathrm{IH}$ patients in the adult group, followed by MsPGN, GML, and EnPGN. Although reports varied by pathological type, the above data show that most IH patients exhibited mild renal pathology, whereas a small percentage had more severe pathological lesions. Experts should thus be cautious, especially when evaluating adult $\mathrm{IH}$ patients, who exhibit a higher IgAN percentage. Renal biopsy should be actively conducted to confirm the pathological type and thus initiate the most effective therapy. As for 
child/adolescent IH patients, although MsPGN accounted for the highest percentage of cases, IgAN still accounted for more than one quarter $(26.32 \%)$ of cases; hence the prognosis for $\mathrm{IH}$ patients is not benign $[14,15,16]$. Renal biopsy should also be considered to achieve early diagnosis and appropriate treatment.

Studies of the relationship between clinical and pathological factors of $\mathrm{IH}$ patients have shown that MsPGN and IgAN are the two most common pathological types among different clinical manifestations. These two types accounted for more than $85 \%$ of cases, where the MGH group was associated with the highest percentage of MsPGN (49.12\%), followed by IgAN, GML, and EnPGN. In the SAP group, $\operatorname{Ig} A \mathrm{~N}$ accounted for the highest percentage (52.61\%) of cases, followed by MsPGN, GML, and EnPGN. These results differed from previous studies $[3,5,17,18,19,20]$ and might be related to differences in the inclusion and exclusion criteria of the various studies, especially proteinuria standards. For example, in the study of Parmar [21], a urinary protein excretion rate $<1 \mathrm{~g} / \mathrm{d}$ was the inclusion criterion, whereas Chow et al. [17] set the proteinuria level at $0.3 \pm 0.1 \mathrm{~g} / \mathrm{d}$. Given that a higher baseline protein excretion rate was selected by Chow and colleagues, the pathological types were more severe than in Parmar's study. In the present study, the patients were grouped according to age. Regardless of the age of the patients, no difference was found in the constituent percentage of pathological types among the different clinical groups. Further analysis of patients with MsPGN revealed that different age groups exhibiting dissimilar clinical manifestations had mild MsPGN. The analysis of patients with IgAN showed that among children/adolescents, Lee grade II IgAN was the most common among IH patients with different clinical manifestations. In the adult group, the MGH group most commonly exhibited Lee grade II IgAN. The SAP group most commonly exhibited Lee grade III IgAN, and pathological classifications were generally more severe than in the MGH group. Microalbuminuria was a sensitive indicator of early renal injury [22]. Our study shows that the detection of microalbuminuria level in IH patients, especially in adult patients, has some clinical significance in determining pathological type.

In summary, IH patients of different age groups exhibited different clinical manifestations and pathological features. The IH patients with different clinical manifestations also had diverse pathological types: the percentage of cases of IgAN, which can lead to progressive renal injury, was relatively high, especially in adult IH patients and SAP-accompanied patients. When IgAN patients exhibited SAP, the severity of the disease was much more serious. Although major nephrology physicians still believe that $\mathrm{IH}$ follows a relatively benign course and biopsy does not affect treatment options or prognosis, our study again illustrates the importance and necessity of early renal biopsy for clinically diagnosed IH. Renal biopsy, which is particularly important for those who have SAP, can help physicians develop a long-term treatment plan and prognosis evaluation.

\section{The authors declare no conflict of interest.}

\section{References}

1. Yamagata K, Takahashi H, Tomida C, et al. Prognosis of asymptomatic hematuria and/or proteinuria in men. High prevalence of IgA nephropathy among proteinuric patients found in mass screening. Nephron 2002; 91: 34-42.

2. Nieuwhof C, Doorenbos C, Grave W, et al. A prospective study of the natural history of idiopathic non-proteinuric hematuria. Kidney Int 1996; 49: 222-225.

3. Shen P, He L, Jiang Y, et al. Useful indicators for performing renal biopsy in adult patients with isolated microscopic haematuria. Int J Clin Pract 2007; 61: 789-794.

4. Liu H, Peng Y, Liu H, et al. Renal biopsy findings of patients presenting with isolated hematuria: disease associations. Am J Nephrol 2012; 36: 377-385.

5. Feng CY, Xia YH, Wang WJ, et al. Persistent asymptomatic isolated hematuria in children: clinical and histopathological features and prognosis. World J Pediatr 2013; 9: 163-168.

6. Chen W, Chen W, Wang H, et al. Prevalence and risk factors associated with chronic kidney disease in an adult population from southern China. Nephrol Dial Transplant 2009; 24: 1205-1212

7. Vivante A, Afek A, Frenkel-Nir Y, et al. Persistent asymptomatic isolated microscopic hematuria in Israeli adolescents and young adults and risk for end-stage renal disease. JAMA 2011; 306: 729-736.

8. Churg J, Bernstein J, Glassock RJ. Lupus nephritis. In: Renal Disease: Classification and Atlas of Glomerular Diseases. Igaku-Shoin, Tokyo 1995; 151-179.

9. Lee SM, Rao VM, Franklin WA, et al. IgA nephropathy: morphologic predictors of progressive renal disease. Hum Pathol 1982; 13: 314-322.

10. Choi IJ, Jeong HJ, Han DS, et al. An analysis of 4,514 cases of renal biopsy in Korea. Yonsei Med J 2001; 42: 247-254.

11. Zhang GJ, Shen Y. Histological categories of asymptomatic hematuria in children analysis of 105 cases. Beijing Medical Journal 2004; 26: 254-256.

12. Mo Y, Chen SM. Pathology and prognosis of children with isolated hematuria. Chin J Obs/Gyne \& Pediatr (Electronic Version) 2005; 2: 82-83.

13. Hall CL, Bradley R, Kerr A, et al. Clinical value of renal biopsy in patients with asymptomatic microscopic hematuria with and without low-grade proteinuria. Clin Nephrol 2004; 62: 267-272.

14. Bergstein J, Leiser J, Andreoli S. The clinical significance of asymptomatic gross and microscopic hematuria in children. Arch Pediatr Adolesc Med 2005; 159: 353-355.

15. Shu KH, Ho WL, Lu YS, et al. Long-term outcome of adult patients with minimal urinary abnormalities and normal renal function. Clin Nephrol 1999; 52: 5-9.

16. Molony DA. ACP Journal Club. Young adults with persistent, asymptomatic, isolated microscopic hematuria were at increased risk for ESRD. Ann Intern Med 2011; 155: JC6-12.

17. Chow KM, Kwan BC, Li PK, et al. Asymptomatic isolated microscopic haematuria: long-term follow-up. QJM 2004; 97 : 739-745. 
18. Kovacević Z, Jovanović D, Rabrenović V, et al. Asymptomatic microscopic haematuria in young males. Int J Clin Pract 2008; 62: 406-412.

19. Kim BS, Kim YK, Shin YS, et al. Natural history and renal pathology in patients with isolated microscopic hematuria. Korean J Intern Med 2009; 24: 356-361.

20. Cai Y, Chen SM. Clinical analysis of 128 cases of isolated hematuria in children. New Medicine 2006; 37: 733-734.

21. Parmar MS. Isolated microscopic haematuria. QJM 2005; 98: 232.

22. Ariceta G. Clinical practice: proteinuria. Eur J Pediatr 2011; 170: $15-20$

\section{Address for correspondence}

Lu Ma

Department of Nephrology

Beidaihe Sanatorium of Beijing Military Area Command

Qinhuangdao 066100, China

tel. +863355360274

fax: +863355360270

e-mail: lumadoc@163.com 